University for Business and Technology in Kosovo

UBT Knowledge Center

UBT International Conference

2016 UBT International Conference

Oct 28th, 9:00 AM - Oct 30th, 5:00 PM

\title{
Introduction to Ships Satellite Tracking Systems
}

Dimov Stojce llcev

Durban University of Technology, ilcev@dut.ac.za

Follow this and additional works at: https://knowledgecenter.ubt-uni.net/conference

Part of the Communication Commons, and the Computer Sciences Commons

\section{Recommended Citation}

Ilcev, Dimov Stojce, "Introduction to Ships Satellite Tracking Systems" (2016). UBT International Conference. 55.

https://knowledgecenter.ubt-uni.net/conference/2016/all-events/55

This Event is brought to you for free and open access by the Publication and Journals at UBT Knowledge Center. It has been accepted for inclusion in UBT International Conference by an authorized administrator of UBT Knowledge Center. For more information, please contact knowledge.center@ubt-uni.net. 


\title{
Introduction to Ships Satellite Tracking Systems
}

\author{
Dimov Stojce Ilcev \\ Durban University of Technology (DUT), Durban, RSA, \\ ilcev@dut.ac.za
}

\begin{abstract}
This paper introduces the current and new Satellite solutions for local and global tracking of ships for enhanced Ship Traffic Control (STC) and Ship Traffic Management (STM) at sea, in sea passages, approaching to the anchorages and inside of seaports. All transportation systems and especially for maritime applications require far more sophisticated technology solutions and equipment for modern Satellite ship tracking than current standalone the US GPS or Russian GLONAS positioning systems. The existing and forthcoming Global Ship Tracking (GST), Satellite Data Link (SDL) and Local Ship Tracking (LST) systems with Space and Ground Segment infrastructures for all three systems are discussed including benefits of these new technologies and solution for improved STC.
\end{abstract}

Keywords: GPS, GLONASS, GEO, GNSS, SAT

\section{Introduction}

A major goal is proposed near-universal use of GNSS of the US GPS and Russian GLONAS infrastructures integrated with Satellite Mobile Communication Systems, which very small units will be able to improve ship tracking, collision avoidance and positioning facilities providing reliable Position, Velocity and Time (PVT) data. The new augmenting system of GNSS are also proposed and projected to enhance Traffic Control Management (TCM) for merchant ships including for enhanced safety and security. As a result of these efforts, new tracking techniques have been projected and developed to utilize Communication, Navigation and Surveillance (CNS) solutions and services for enhanced Ship Traffic Control (STC) and management for improved safety and security in commercial maritime transportation.

In order to meet the requirements for better CNS solutions of ocean sailing, approaching to seaports and inside of seaports it is also proposed development of Global Ship Tracking (GST) including Satellite Automatic Identification System (S-AIS) solutions. Thus, these new technologies will cover the entire African Continent and the rest of the world for ocean and coastal navigation and can improve tracking and determination of all types of ships. The new GST and other existing solution for determination will improve the basic GPS and GLONASS facilities and allow to these GNSS networks to be utilized with Satellite transceivers as a primary means of tracking of ships and all land vehicle movements in the seaports via Maritime Traffic Control Centres (TCC). Proposed satellite tracking systems may be used in all possible applications for determination spatial coordinates such as position, speed and navigational status of target objects, which via GNSS equipment may provide PTV data for maritime and all transportation applications.

These systems mainly are necessary for improved collision avoidance of ships especially in areas with heavy traffic moving such as sea channels, approaching to anchorages and inside of seaports. Using standalone GPS or GLONASS data these systems can provide speed and position of ships only. However, integrated with GNSS units and some sensors, these systems are also capable to control main parameters of oceangoing ships such as continuous position control, 
Book of Proceedings

International Conference on Computer Science and Communication Engineering

mileage, consumption of fuel, frequently can transmit position of ships and other parameters. The position data can be used in case of ships grounding, hijacking or emergency abandoned ships and for eventual Search and Rescue (SAR) actions. Appearance and implementation of these systems may provide very important contributions in enhanced safety and security for all type of ships and according to the International Maritime Organization (IMO) to improve distress and SAR operations.

The tracking systems are working without man intervention in order to prevent human errors. Continuous increasing of ships transport is augmenting the necessity per explores the emerging safety and security solutions for maritime CNS systems and especially for tracking and data messaging facilities. These new tracking techniques consider the consequences of a global satellite communication framework supporting asynchronous messaging of navigation data that can be used to enhance the basic GST and AIS capability. In that manner, the analyzed modern satellite GST application can be pursued within the standardization process or independently developed with attention to compatibility with existing radio systems.

The GAT system is projected by author of this paper, which is integrating GPS Receiver (Rx) and Iridium or Inmarsat satellite transceivers with antennas installed onboard ships. Thus, the tracking unit has to be installed discrete onboard ships secret location, which solution has to protect accidental or forced its shutdown $[1,2]$.

\section{Development of GST Networks and Equipment}

The scenario of Satellite Asset Tracking (SAT) is system employing the GNSS subsystem of US GPS and Russian GLONASS to provide free of charge Position (PTV) data to different users at sea, on the ground and in the air. This PTV data can receive ships, land vehicles and aircrafts via onboard GPS or GLONASS. If Receivers (Rx) and used in navigation purpose, which Inmarsat network is depicted in Figure 1GPS or GLONASS Rx is integrated with Satellite Transceiver, Rx and Transmitter (Tx) in an integrated satellite unit with both antennas known as SAT, it will be possible to provide frequently transmission of PTV data via Geostationary Earth Orbit (GEO) and Non-GEO spacecraft through Ground Earth Station (GES) and Internet to the Control and Operations Centres.

Because of many incidents in past time with difficulties of searching ships in disaster and for improvement of collisions avoidance of ships the author of this paper is proposing new tracking and determination solutions via Satellite CNS and determination systems known as Global Ship Tracking (GST). Similar to the Long Range Identification and Tracking (LRIT) new and more advanced GST solution contains the shipborne GST information transmitting equipment, such as integrated GPS or GLONASS Rx and GEO pr Non-GEO, such as Inmarsat or Iridium satellite transceivers, namely Transmitter and Receiver (Tx/Rx). 


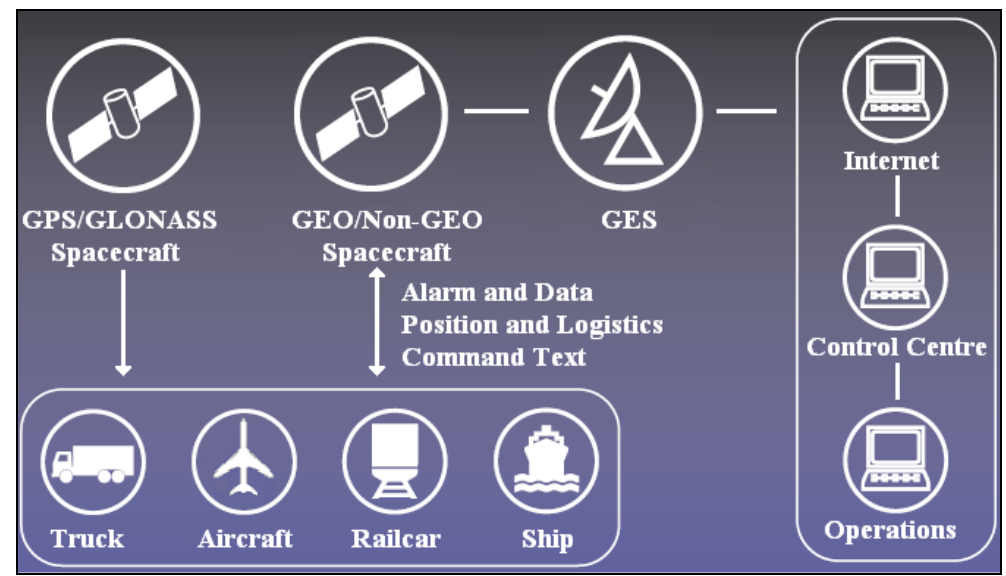

Fig. 1. Configuration of SAT via GNSS and GEO/Non-GEO Satellites [2]

Because of many incidents in past time with difficulties of searching ships in disaster and for improvement of collisions avoidance of ships the author of this paper is proposing new tracking and determination solutions via Satellite CNS and determination systems known as Global Ship Tracking (GST). Similar to the Long Range Identification and Tracking (LRIT) new and more advanced GST solution contains the shipborne GST information transmitting equipment, such as integrated GPS or GLONASS Rx and GEO pr Non-GEO, such as Inmarsat or Iridium satellite transceivers, namely Transmitter and Receiver (Tx/Rx).

The shipborne GST onboard equipment receives GNSS determination signals from GPS/GLONASS spacecraft (1) and sends PTV tracking messages of position (2) via GEO satellite to Ground Earth Station (GES (3) of Satellite Communication and Application Service Providers (Internet) to the TCC processor (4), shown in Figure 2.

The current LRIT and new proposed GST have the same services explained above and in addition both can provide pulling navigation data of any ship from TCC sites. The difference between them is that LRIT is not determined to provide that some ship can receive navigation data of near by ships for enhanced collision avoidance. Thus, the red lines highlighted in Figure 2 can be used for pulling service and what is more important for sending to any ship on his request the navigation data of adjacent ships in the same sea area.

Onboard oceangoing ships can be installed many of satellite tracking equipment already designated for SAT onboard all mobiles such as vehicles, trains, containers and aircraft, but with simply modification for very harsh weather and severe sea conditions. This equipment has to suitable for:

1. Possibility for installation onboard each mobile including ship, and some have to carry 3 to 5 years batteries, so to work properly even when ship is in emergency situations without any power supply;

2. Pre-programming for different requirements and to send GPS location and other data on predefined intervals via any GEO or Non-GEO satellite systems to shore host application or can be integrated with a mapping application; 
Book of Proceedings International Conference on Computer Science and Communication Engineering

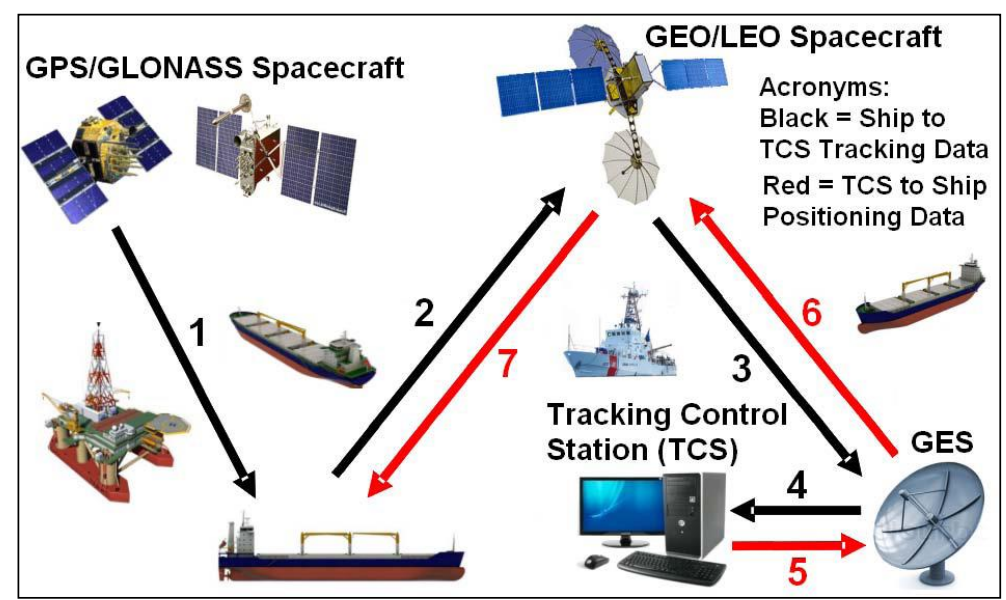

Fig. 2. Configuration of GST via GNSS and GEO/Non-GEO Satellites [2]

3. Pulling facilities at shore TCS and getting position of any desired ships in vicinity; and

4. Sending positioning messages from TCS to all ships requesting these navigation data of adjacent ships.

As stated before, current LRIT system is not projected to do this very important service for collision avoidance of ships. It is important to express that LEO Globalstar and Orbcomm satellite systems are providing both simplex and duplex (two-way) satellite transmission. Duplex satellite system is able to provide sending and receiving of GST data, while simplex system only can enable receiving facilities of navigation data for adjacent ships. However, the third LEO Iridium and GEO Inmarsat mobile satellite systems are providing duplex service only.

At present the following four satellite operators are providing satellite constellations for SAT service:

1. Inmarsat Geostationary Earth Orbit (GEO) satellites for providing coverage up to $75^{0}$ North and South;

2. Iridium Big Low Earth Orbit (LEO) satellites for full global coverage because of intersatellite links;

3. Globalstar Big LEO with limited coverage depending on distributed number of Gateways; and

4. Orbcomm Little LEO with limited coverage depending on distributed number of Gateways $[2,3,4,5]$.

\subsection{Inmarsat GST Equipment and Data Network}

Inmarsat was established as not-for-profit company in 1979 as the International Maritime Satellite Organization (Inmarsat) initially for development maritime satellite communications. It began trading in 1982 via GEO satellite constellation for almost global coverage, but is not covering both poles. Afterwards Inmarsat started with development service for land (road and rail) and aeronautical applications. 


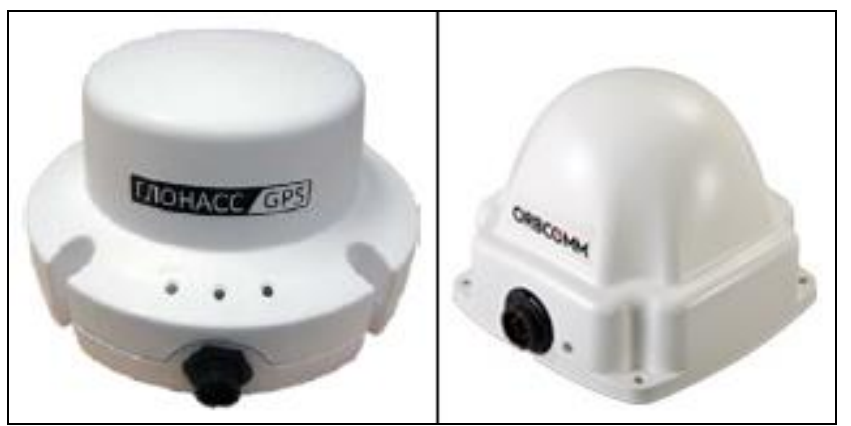

Fig. 3. Two Generations of Inmarsat-D+ and IsatData Pro Systems [4]

Today Inmarsat is transformed in Private operating company providing duplex satellite communication at the following Radio Frequency (RF) bands: $1.6 / 1.5 \mathrm{GHz}$ of L-band (Service Link) and at 6.4/3.6 GHz of C-band (Feeder Link).

The former-Inmarsat D+ transceiver with successor IsatData and IsatM2M are developed on basis of Inmarsat-C standard, which are the best solution for GST via satellites including AIS. It is able to transmit and receive GST data anywhere via Inmarsat satellite constellation and is ideal for determination, asset tracking and security in navigation, fleet management and SCADA applications. Besides, the GST transceiver is low powered by onboard ships and batteries power supply with possibility to work even if ships is grounded somewhere without main power. This unit is integration of GPS Rx and Satellite Transceiver with both antennas.

Features of these units are two-way messaging up to 25-byte message data size from terminal, up to 100-byte message size to terminal. Fast message delivery in 1 minute to terminal and rapid response in 10 seconds from terminal. This unit can be integrated with GPS Rx providing speed and position data. With external additional sensors it monitors consumption of fuel, mileage, temperatures and etc.

Recently Inmarsat has developed IsatM2M and IsatData as two-way Short Burst Messaging (SBM) service that enables a wide range of SAT and SCADA (M2M) solutions for tracking and monitoring fixed or mobile assets on a global basis, whether at sea, on the land or in the air. The new generation IsatM2M and IsatData Pro satellite telematics is based on Inmarsat D+ standard, offering faster data forwarding rates, quicker responses to polling requests and shorter time to first transmission. Inmarsat offers two models of unpackaged satellite SAT of SkyWave (today Orbcomm) producer:

1. Inmarsat-D+ DMR-800L Terminal - This Inmarsat-D+ satellite transceiver is dedicated for many SAT and GST solutions via both GPS and GLONASS navigation signals, which device is depicted in Figure 3 (Left). This device provides a flexible, unpackaged assembly of satellite transceiver integrated with GPS or GLONASS receiver, which uses in-unit or separate satellite/GNSS antenna and discrete input and output feeds. In addition, it contain built-in processor/controller board allows the unit to work as a simple modem and to interface a set of sensors and actuators. This mobile terminal is able to support GST powered by ship power supply or can be as alternative easily packaged with long life batteries to provide satellite communication service when ship has not own power supply. 
Book of Proceedings

International Conference on Computer Science and Communication Engineering

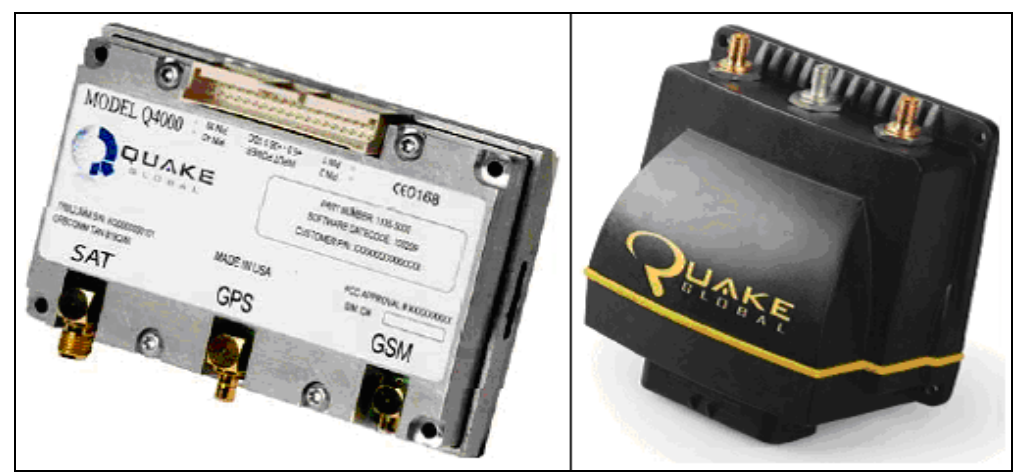

Fig. 4. Iridium Satellite Trackers [4]

2. Inmarsat IsatData Pro IDP 600 - This modern IDP 600 series terminals is fully programmable and environmentally sealed that uses two-way GEO Inmarsat IsatData Pro satellite service to provide visibility and communications with people and fixed or mobile equipment even in the world's harshest environments, which device is shown in Figure 3 (Right). In particular, IsatData Pro is the fastest low-data rate satellite communications equipment especially suitable for vessel tracking and management, such as to enhance maritime safety and Guide Rescue Operations Simplify; to provide Vessel Monitoring System (VMS); to reduce vessel fuel costs and monitor engine performance; and to monitor vessel performance and reduce paperwork. This tracking device, as stated above, is integration of Inmarsat satellite transceiver and GPS or GLONASS (or both) Rx. In fact, if this device is implemented as GST equipment onboard ships and connected to special GST Network will be able to provide satellite tracking and detection of missed or hijacked ships by pirates, and what is very important as well as to provide enhanced service for collision avoidance $[3,4,5]$.

\subsection{Iridium GST Equipment and Data Network}

The Iridium is situated in a near-polar Low Earth Orbit (LEO) orbit at an altitude of $780 \mathrm{~km}$. They circle the Earth once every 100 minutes travelling at a rate of about $26,856 \mathrm{~km} / \mathrm{h}$. Thus, each satellite is cross-linked (inter-satellite service) to four other satellites, namely two satellites in the same orbital plane and two in an adjacent plane.

The Iridium Big LEO satellite constellation consists in 66 operational satellites and 14 spares orbiting in a constellation of six polar planes, providing real global coverage including both poles and roaming via 48 spot overlapping beams and the diameter of each spot is about $600 \mathrm{~km}$.

Iridium as a real global satellite operator that provides two-way voice and data communication service including SAT for all mobile applications via RF links at $1621.35-1626.5 \mathrm{MHz}$, feeder links at 29.129.3 GHz of Ka-band (uplink) and at: 19.4-19.6 GHz of K-band (downlink) and satellite cross-link or inter-satellite link at $23.1823 .38 \mathrm{GHz}$ of Ka-band. Among the rest, the Iridium satellite network is providing SAT service for all fixed and mobile applications including maritime with the following satellite tracking devices: 


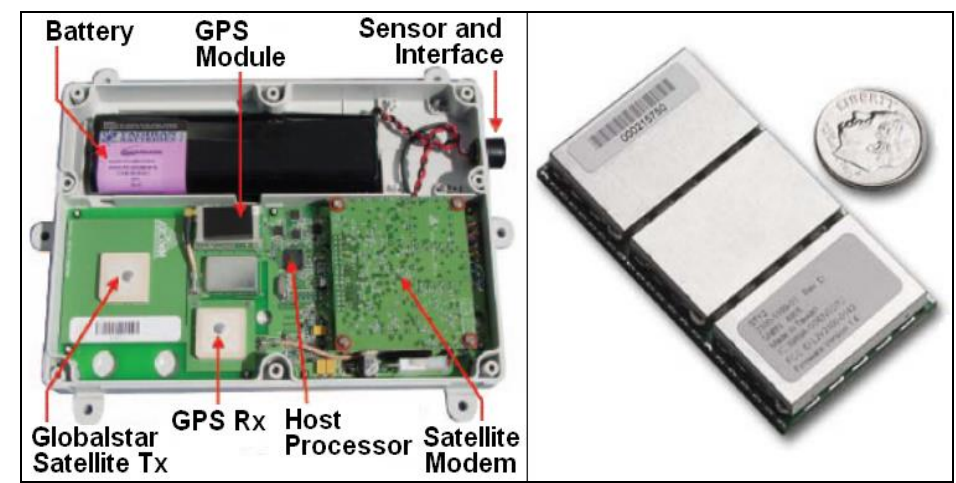

Fig. 5. Configuration of Globalstar GST Equipment [6]

1. Quake Q4000 Terminal - Though the Iridium Q4000i is a small enough to fit in hand produced by the US Company Quake. It is a two-way rugged industrial grade modem that can combines dual-mode operability over multiple satellite constellations and GSM terrestrial networks with GPS into a versatile, all-in-one mobile and remote asset tracking solution. The same SAT unit can be optionally supplied for service over Inmarsat, Globalstar and Orbcomm integrated with GPS Rx and with optional GSM cellular service. Technically this is a Short Burst Data (SND) transceiver designed for use as basic unit for many trackers using the Iridium Network, which is depicted in Figure 4 (Left). This unit can be used for ocean ships and container tracking and as well as for land vehicles and aircraft tracking.

2. Quake Q-Pro Multipurpose Tracker - This Iridium transceiver is a small (119.2x119.4x57.6 mm and 390.6 grams) integrated Iridium, Globalstar, Orbcomm and GSM satellite modem with GPS receiver, which is illustrated in Figure 4 (Right). It can be used for GST including for containers, trucks, trains and aircraft tracking and monitoring $[3,4,6,7]$.

\subsection{Globalstar GST Equipment and Data Network}

The US Loral Space and Communications, with Qualcomm Incorporation developed the concept of Globalstar system at a similar time to Iridium. Globalstar gained an operating license from the USA FCC in November 1996.

The first launch of four Globalstar satellites occurred in May 1998 building space segment of 48 Big LEO spacecraft. The system uses Code (CDMA) and Frequency Division Multiple Access (FDMA) methods with an efficient power control technique, multiple beam active phased array antennas for multiple access and frequency reuse of voice and data transmission.

Globalstar is not providing inter-satellite links and therefore needs a number of GES terminals worldwide. Otherwise, this system started to provide coverage for South Africa in 2015. Globalstar is providing service for users via satellite at $1.610-1.621 \mathrm{GHz}$ (uplink) and at 2.483$2.500 \mathrm{GHz}$ (downlink) and from satellite to GES at 5.091-7.055 GHz (feeder link).

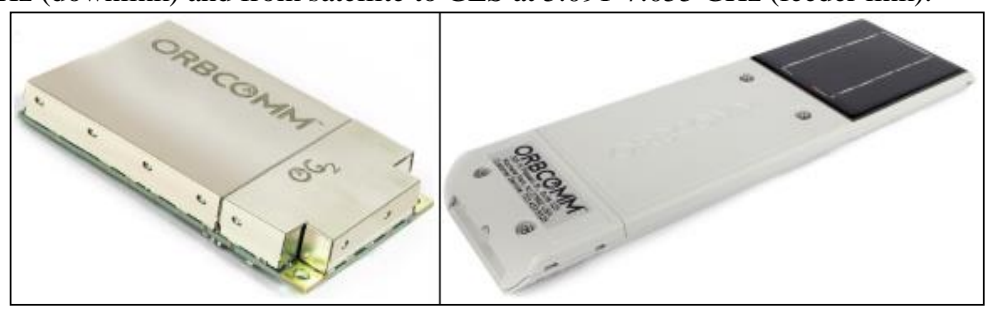

Fig. 6. Configuration of Orbcomm GST Equipment [6] 
Book of Proceedings

International Conference on Computer Science and Communication Engineering

The Globalstar satellite configuration for data communication, illustrated in Figure 5 (Left) is using Big LEO Globalstar satellite network for simples (one-way) data transmissions. The main parts of SAT device are GPS Rx for receiving of GPS tracking data and Satellite Tx for sending PVT data to the TCC via GEO or Non-GEO satellites. This device may be powered by onboard power supply or via own long-term batteries. In Figures 5 (Right) is depicted another samples SAT of former-Axonn satellite terminal of very small sizes $[3,4,6,8]$.

\subsection{Orbcomm GAT Equipment and Data Network}

The Little LEO Orbcomm satellite communication system is a wide area packet switched and two-way data network providing satellite communication, tracking and monitoring services globally for fixed and mobile assets via 36 LEO satellites. Since 2012 Orbcomm is covering Southern Africa offering messaging services via small GPS/Orbcomm satellite trackers even for GST on VHF-band at 148.0-150.05 MHz (Service/Feeder uplink) and at 137.0-138.0 MHz (Service/Feeder downlink). The Orbcomm operator is also developing system that will provide Satellite Automatic Identification System (S-AIS) for broadcast and ship's identification, position and other critical data for improving safety and security at sea. The Orbcomm network is providing GST and other container and mobile employing the following units:

1. Orbcomm OG2-GPS Modem - This SAT unit delivers connectivity over the LEO Orbcomm VHF satellite network for marine, heavy equipment, transportation, agricultural and other markets, which is depicted in Figure 6 (Left).

2. Orbcomm GT 1100 Modem - This small satellite data unit powered by solar rechargeable batteries enables full control of mobile assets and containers, shown in Figure 6 (Right) [3, 4, 6, 9].

\section{Global Ships Tracking (GST) Network}

The LRIT satellite transceiver system is new compulsory equipment onboard ships established by IMO for vessel tracking worldwide. This system consists of the shipborne data/information transmitting satellite equipment (similar to above stated), the Communication Service Provider(s), the Application Service Provider(s), the LRIT Data Centre(s), including any related Vessel Monitoring System(s), the LRIT Data Distribution Plan and the International LRIT Data Exchange.

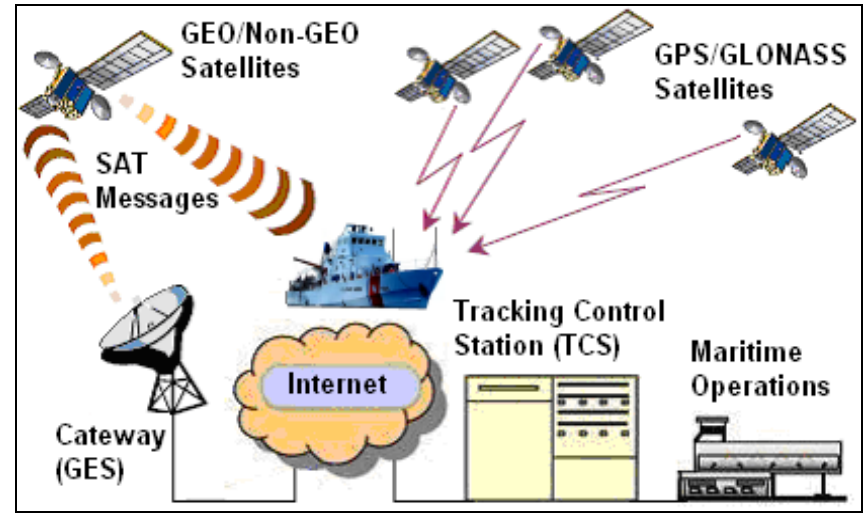

Fig. 7. Global Ship Tracking (GST) Network [2] 
Introduction to Ships Satellite Tracking Systems

As stated earlier, disadvantages of LRIT system that cannot transmit navigation data of adjacent ships on request of any ship sailing in certain sea area for collision avoidance, and that LRIT is not able to provide tracking of missing or hijacked ships.

The proposed GST solution is able to provide all service as LRIT including to provide tracking of missing and hijacking ships, and to determine positions of all ships in vicinity of ship requesting this data for collision avoidance as the best for vessel tracking worldwide.

In Figure 7 is introduced new and simplest concept of GST for worldwide vessels tracking and monitoring. In fact, using satellite links of GEO or Non-GEO satellites any ship is able to send automatically its PVT data, provided by GPS or GLONASS satellites, via Gateway (GES) and Internet to the Tracking Control Station (TCS) and Ships Operations. In opposite direction Control Centre can provide pulling navigation data from any ship, and what LRIT cannot do, Control Centre can send to any ship on his demand position data of all ships in his vicinity for collision avoidance and enhanced safety and security at sea $[2,6,8,10]$.

\section{Satellite Data Link (SDL) Network}

The GEO and Non-GEO (LEO) satellite constellations can provide Satellite Data Link (SDL) or AIS (S-AIS) for onboard broadcast solution that transmits a ship's identification, position and other critical data that can be used to assist in ships navigation and tracking facilities for improvement maritime safety and security. The SDL system can provide transmission of Short Burst Messages (SBM) between mobile stations or terminals with GES, Control Centre and Maritime Operation, which is illustrated in Figure 8.

In mobiles, such as ships and surface vehicles in seaport, can be installed special satellite transponders or already stated satellite tracker devices. Mobile transponders can operate autonomously inside the coverage of certain Gateway (GES). The SDL transponder can support the similar services that provide Radio VDL4, but if is using Iridium transponder will be able to provide global coverage including both Poles.

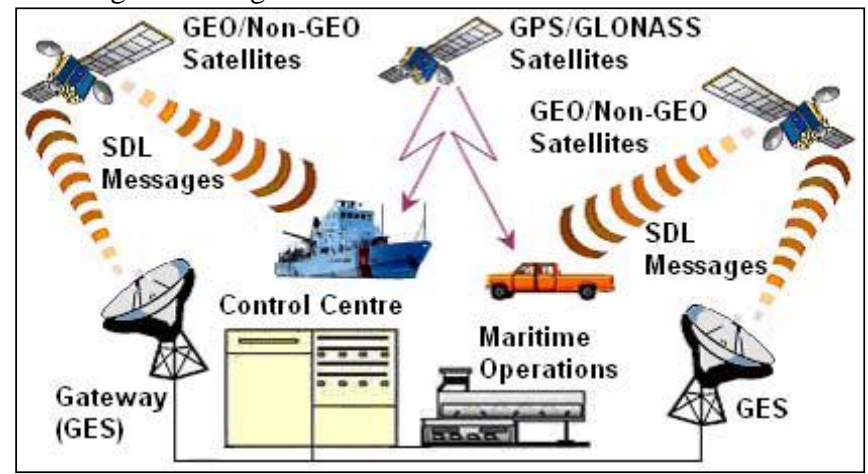

Fig. 8. Satellite Data Link (SDL) Network [2]

The SDL transponder allows captains onboard ships and maritime traffic controllers to receive all vessels traffic data in ocean or coastal navigation, approaching to seaport and inside of harbours including vehicle movements with the highest possible precision. The receiving SDL units can receive all SDL messages and process them in sophisticated processor. The receiving SDL messages can be transfer and monitor on special display looks like radar screen. In the same way will be processed and monitored GST data in TCS. The SDL system may drastically improve safety and security at sea and in seaport area as well. 
Book of Proceedings

International Conference on Computer Science and Communication Engineering

The Gateway (GES) terminals can cover satellite systems, such as Inmarsat, Globalstar, Iridium or Orbcomm networks worldwide and easily interface with other surveillance systems through the standardized Asterix protocol, which will enable a complete tracking surveillance picture at the seaport derived from several sources. The GES terminal and a ground network will provide increased functionality and capability for wide area coverage of advanced STC Monitoring and Management.

The functionality of the GES terminal is tailored to the specific service applications by its software configuration. Therefore, in Figure 8 is presented that ships and vehicle SDL terminals receive GPS or GLONASS positioning (PVT) signals and automatically send this data via GEO/Non-GEO satellites and GES to Control Centre and Ships Operations. In vice versa direction Control centre can send instructions to ships how can move more safely at sea and seaports and to vehicles in seaports only. Therefore, to get SDL service working, each ship and ground vehicles have to be equipped with SDL transponders or satellite communication devices and in such a way will be able to send and receive SBD or High Speed Data (HSD) for CNS and collision avoidance purposes $[2,9,11]$.

\section{GNSS Augmentation SDL (GASDL) Network}

The Regional Satellite Augmentation System (RSAS) of the US GPS or Russian GLONASS, both integration part of GNSS-1 infrastructure is a combination of ground and space equipment dedicated to provide augmentation of standard GPS or GLONASS signals, which infrastructure is illustrated in Figure 9. The functions being provided by RSAS are:

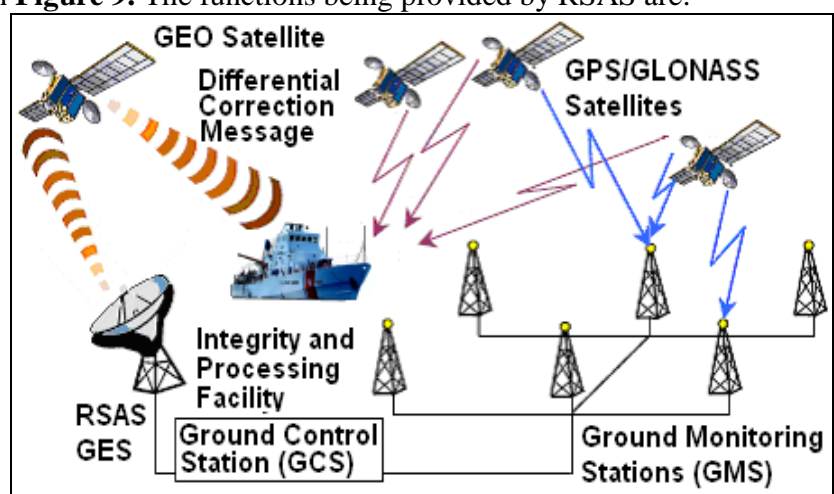

Fig. 9. GNSS Augmentation SDL [2]

1. Differential corrections are determined to improve GNSS signal accuracy;

2. Integrity monitoring is predisposed to ensure that errors are within tolerable limits with a very high probability and thus ensure safety; and

3. Ranging is proposed to improve availability.

The numbers of Reference Stations (GMS) are receiving not augmented signals of GPS or GLONASS satellites, processing and forwarding this data to Master Station (GCS). The GCS terminals provide processing of GNSS data to determine the differential corrections and bounds on the residual errors for each monitored satellite and for each area. Therefore, GCS terminal is providing determination of the clock, ephemeris and ionospheric errors (ionospheric corrections are broadcast for selected area) affected during propagation. The corrections and integrity information from the GCS terminal are then sent to each RSAS GES and uplinked to the GEO Satellites. Thus, these separate differential corrections are broadcast by RSAS GES through GEO 
Introduction to Ships Satellite Tracking Systems

satellite data link via GNSS transponder at the same frequency used by not augmented GPS receiver.

For instance, augmented GPS Rx is receiving augmented signals of GPS satellite and determining more accurate position of ships. Not augmented GPS Rx can also receive augmented signals if is provided an adequate software or hardware. The most important stage in this network is to provide technical solution that augmented position of aircraft can be sent automatically via SDL or voice to GES and Control Centre. Finally, these positioning signals can be processed by special processor and displayed on look like radar display, whish traffic controller is using for STC and management for enhanced ship traffic control and improved collision avoidance in certain monitoring sea area $[2,8,12]$.

\section{Conclusions}

In this paper were described and shown some quite spread, embedded and sustain systems for maritime satellite tracking applications very important for oceangoing ships, crew and passengers safety and security in all phase of ocean, coastal and passage navigation.

At this point, every ship operators can deploy any satellite Ship Earth Stations (SES) and tracking equipment according to the IMO recommendations and Safety of Lives at Sea (SOLAS) regulations and cost effective sense. In that manner, the major point of GST network is to find out the best solutions for more reliable global maritime satellite communications, tracking, determination, monitoring and enhanced collision avoidance system with priorities of ships safety and security.

Today, Inmarsat GEO satellite operator for mobile satellite communication is only professional system providing near global coverage up to $75^{\circ}$ North and South. In any case, with regards to available ocean areas coverage this system and equipment can be used for any types of oceangoing ships in any stage of navigation.

Presently, ships are not sailing in Arctic Ocean, but Russian government is proposing these routes, where can be used HF communication systems instead. However, Iridium satellite operator as not professional system is providing full global coverage thanks to inter-satellite links, however Globalstar and Orbcomm LEO have limited coverages.

Therefore, the future of maritime and other mobile satellite communication is combination of GEO, LEO and other orbits, like Medium Earth Orbit (MEO) and High Elliptical Orbit (HEO) in so called Hybrid Satellite Orbits (HSO), which can provide a professional service globally and over Arctic Ocean.

\section{References}

1. Ilcev D. S., "Global Mobile Satellite Communications for Maritime, Land and Aeronautical Applications", Springer, Boston, 2005.

2. lcev D.S., "Global Mobile Communications, Navigation and Surveillance (CNS)", DUT, Durban, 2012.

3. Group of Authors, Web Sites: (www.inmarsat.com; www.iridium.com; www.globalstar.com; www.orbcomm.com), 2016.

4. Ilcev D. S., "Global Aeronautical Communications, Navigation and Surveillance (CNS)", Volume 1 \& 2, AIAA, Reston, 2013.

5. Kantor L.Y. \& Others, "Sputnikovaya svyaz i problema geostacionarnoy orbiti”, Radio i svyaz, Moskva, 1988. 
Book of Proceedings

International Conference on Computer Science and Communication Engineering

6. Ilcev D. S., "Global Ship Tracking, Automatic Identification System and Determination”, Presentation, Space Science Centre (SSC) and Postgraduate Studies, DUT, 2011.

7. Bendisch J., Hoffmann H., Wieneke T., and Niemöller H.G., "Aeromission - An Airborne System for Maritime Surveillance", Aerodata AG, Braunschweig, Germany, 2005.

8. Stacey D., "Aeronautical Radio Communication Systems and Networks”, John Wiley, Chichester, 2008.

9. Maral G. \& Others, "Satellite Communications Systems”, Wiley, Chichester, 2009.

10. Myron K. \& Fried W. R., “Avionics Navigation Systems” Wiley, 1997.

11. Ilcev D.S. "Satellite CNS for Maritime Transportation Augmentation System (MTAS)", CriMiCo Conference, IEEE CFP09788, Sevastopol, Russia, 2009.

12. Ilcev D.S., "Maritime Communications, Navigation and Surveillance (CNS)", Manual, DUT, Durban, 2010. 